\title{
Ensayos Económicos
}

La contundente justificación para una normativa de apalancamiento más sólida y efectiva para los bancos

Anat Admati

Un análisis de los desequilibrios del tipo de cambio real argentino bajo cambios de régimen Daniel Aromí y Marcos Dal Bianco

La reforma de la Reserva Federal de 2008: ¿La oferta de dinero es endógena o exógena? Guillermo Gigliani

El concepto de probabilidad en la obra de Lord Keynes Alberto Landro 


\title{
La contundente justificación para una normativa de apalancamiento más sólida y efectiva para los bancos
}

\author{
Anat Admati* \\ Universidad de Stanford
}

\section{Resumen}

El exceso de apalancamiento (endeudamiento) en los bancos representa un peligro público y distorsiona el funcionamiento de la economía. Sin embargo, las reformas que se proponen actualmente sólo retocan las regulaciones anteriores, las cuales no lograron garantizar la estabilidad financiera. Este documento analiza las fuerzas que han llevado a esta situación, algunas de las cuales parecen haber sido mal interpretadas. Los beneficios para la sociedad de exigir que las instituciones financieras utilicen significativamente más capital propio que en el statu quo son grandes, mientras que los costos son totalmente privados debido a la capacidad de los bancos para transferir una parte de los mismos a los demás cuando se financian con deuda. Sin realizar un análisis cuantitativo, se proponen mejoras a las normas y cómo podrían implementarse.

Clasificación JEL: G01, G21, G28, G32.

Palabras clave: apalancamiento, bancos, crisis financieras, estructura de financiamiento, normas bancarias, requisitos de capital.

\footnotetext{
* Este documento fue presentado en las Jornadas sobre el Análisis Costo-Beneficio de las Normas Financieras realizada en la Universidad de Chicago, el 18 de octubre de 2013. Agradezco a Yakov Amihud, Martin Hellwig, Paul Pfleiderer, Eric Posner y a un árbitro anónimo por sus útiles comentarios. Las opiniones expresadas en el presente trabajo no se corresponden necesariamente con las del BCRA o sus autoridades. Email: admati@stanford.edu.
} 


\title{
The Compelling Case for Stronger and More Effective Leverage Regulation in Banking
}

\author{
Anat Admati \\ Stanford University
}

\section{Summary}

Excessive leverage (indebtedness) in banking endangers the public and distorts the economy. Yet current and proposed regulations only tweak previous regulations that failed to provide financial stability. This paper discusses the forces that have led to this situation, some of which appear to be misunderstood. The benefits to society of requiring that financial institutions use significantly more equity funding than the status quo are large, while any costs are entirely private, due to banks' ability to shift some of their costs to others when they use debt. Without quantitative analysis, I outline improved regulations and how they can be implemented.

JEL: G01, G21, G28, G32.

Keywords: banking regulation, banks, capital ratios, financial crises, funding mix, leverage. 


\section{Introducción}

Las buenas decisiones requieren un equilibrio entre los beneficios y costos de las posibles iniciativas. Una correcta evaluación del impacto de las normas debe ponderar los diversos costos y beneficios para la sociedad. Las buenas normas compensan los costos privados de los afectados por ellas con beneficios significativamente mayores para los demás.

La crisis financiera de 2007-2009 puso de relieve la fragilidad del sistema financiero y el gran daño que puede ocasionar. Esta fragilidad se exacerba en gran medida por el hecho de que los bancos utilizan muy poco capital (dinero de sus propietarios y accionistas) para financiar sus inversiones. Exigir a los bancos que utilicen más capital permitiría generar un sistema financiero más estable, que sirva mejor a la economía y de manera consistente, al tiempo que resulte menos propenso a los ciclos de auge, caída y crisis.

Los banqueros y otros, a menudo, argumentan que los beneficios de requerir significativamente más fondos de capital deben ser contrastados con los costos de tales requisitos, y alegan que las reformas recientes y en curso son contundentes. Sin embargo, el costo de un aumento significativo de los requerimientos de capital en relación con el statu quo es enteramente privado, debido a la capacidad de los bancos para transferir una parte de los mismos a los demás. Además, las reformas realizadas hasta ahora representan sólo retoques a las fallidas regulaciones anteriores y mantienen un sistema financiero peligroso e imprudente que distorsiona la economía.

En este trabajo se resumen las cuestiones clave para evaluar las normas que requeriría que las instituciones financieras utilicen más capital para financiar sus actividades. El trabajo se basa en gran medida en Admati, DeMarzo, Hellwig y Pfleiderer, abreviado como ADHP $(2013,2014)$, que incluye una revisión detallada y un análisis de los aspectos económicos de la financiación y el apalancamiento de los bancos; y Admati y Hellwig, abreviado como AH (2013a, b, 2014), que se dirige a un público más amplio y que también discute la estructura de las normas y de la economía política relacionada con la banca.

Siguiendo AH (2013a), también se motiva y esboza una propuesta concreta para mejorar la normativa, y se describen los pasos para su implementación. La propuesta no se basa en un análisis cuantitativo de los costos comparativos o en 
una evaluación empírica precisa de las propuestas alternativas. Se considera que no existe ningún modelo que pueda captar adecuadamente los costos comparativos relevantes de ésta u otras propuestas en comparación con el statu quo. Sin embargo, los beneficios de la propuesta, aunque no puedan medirse con precisión, parecen significativos.

El trabajo se organiza de la siguiente manera. La Sección II discute la importancia y los beneficios de una normativa que reduzca aún más el apalancamiento en el sector bancario. La Sección III explica las fuerzas económicas fundamentales que hacen que esa normativa sea esencial. La Sección IV explica por qué algunos de los argumentos relacionados con los costos de incrementar los requisitos de capital representan falsos costos comparativos. En la Sección $V$ se motivan y esbozan los pasos para una propuesta específica que mejore la normativa. La Sección VI ofrece una breve discusión sobre el desafío político, que es una razón clave del fracaso de las normas y de su reforma hasta el momento. La Sección VII concluye con comentarios adicionales sobre las propuestas de reforma financiera.

\section{Los beneficios de reducir el apalancamiento en los bancos}

El apalancamiento financiero se refiere a la combinación de financiamiento de un negocio o empresa entre endeudamiento y fondos de los propietarios o accionistas. Con la promesa de hacer pagos específicos de deuda en momentos específicos, los deudores crean apalancamiento que magnifica el riesgo. Cuando las inversiones realizadas con los fondos prestados salen mal, el endeudamiento puede dar lugar a dificultades financieras, a la insolvencia o al default, lo que puede tener profundas implicancias para los deudores, sus acreedores y posibles terceros. ${ }^{1}$ Este lado oscuro del endeudamiento es particularmente fuerte en el sector bancario, pero los banqueros no lo experimentan de la misma manera que otros prestatarios.

Una empresa fuertemente apalancada es similar a una familia que se compra una gran casa con sólo un pequeño pago al contado. El pago al contado puede asimilarse a un capital accionario inicial que fluctúa junto con el valor de la propiedad, ya que el endeudamiento permanece fijo. Si el valor de la propiedad

${ }^{1}$ Ver AH (2013a, Capítulo 2) para ampliar sobre apalancamiento y riesgo. El "lado oscuro del apalancamiento" se discute en el Capítulo 3. 
cayera lo suficiente su dueño/a podría encontrarse con que la deuda hipotecaria sea mayor que el valor de la casa. Análogamente, una empresa puede entrar en dificultades o tornarse insolvente si su deuda se aproxima o alcanza un valor superior al de sus activos.

Una carga excesiva de deuda aumenta la probabilidad de incumplimiento, lo que puede introducir costos irrecuperables que agoten el valor de los activos. Incluso antes de un incumplimiento, el alto endeudamiento crea distorsiones e ineficiencias debido a los conflictos de interés entre deudores y acreedores. Deudores en dificultades o insolventes pueden subinvertir en proyectos rentables debido al llamado "peso del sobreendeudamiento", al evitar inversiones que no generan suficientes ganancias para los prestatarios, pero que podrían aumentar el valor de los activos de todos los inversores, incluidos los acreedores. Al mismo tiempo, prestatarios sobreendeudados pueden realizar inversiones riesgosas que los beneficien y que perjudiquen a los acreedores, aun cuando el daño a los acreedores sea mayor que la ganancia del deudor, lo que reduce el valor combinado de sus derechos. Los costos de quiebra y las distorsiones de las dificultades financieras y la insolvencia son más intensos cuanto mayor es la dependencia al endeudamiento.

Muchas instituciones financieras atravesaron dificultades financieras o se volvieron insolventes en 2007. La quiebra de Lehman Brothers en septiembre de 2008, que tuvo enormes efectos dominó en todo el mundo, expuso la fragilidad del sistema financiero global. Esta fragilidad financiera se debió al alto nivel de apalancamiento de las instituciones financieras, su opacidad, el uso intensivo de financiamiento con deuda de corto plazo, y el alto nivel de interconexión del sistema. Todos estos elementos crearon corridas y contagio cuando los precios de las viviendas cayeron y los propietarios endeudados comenzaron a incumplir sus compromisos de deuda, un shock que en sí mismo no era grande en relación al tamaño de la economía mundial. ${ }^{2}$

Para evitar un colapso total del sistema, los bancos centrales y los gobiernos otorgaron a la banca créditos extraordinarios, garantías y rescates. Hoy en día, cientos de millones de personas todavía están sintiendo el impacto de la grave recesión que la crisis financiera generó. Mientras que la mayoría de las instituciones financieras evitaron el incumplimiento y fueron capaces de cumplir totalmente con sus acreedores, los propietarios de viviendas con problemas

${ }^{2}$ Para una explicación del mecanismo de contagio, ver AH (2013a, Capítulo 5). 
financieros recibieron relativamente poco alivio dado que los prestamistas no fueron obligados a reestructurar las hipotecas y prefirieron no hacerlo en muchos casos.

En el período previo a la crisis financiera, el patrimonio neto de muchas instituciones representó menos del $3 \%$ de sus activos totales, y en algunos casos ratios tan bajos como $1 \%{ }^{3}$ Muchas de esas instituciones se volvieron insolventes o fueron rescatadas debido a que las inversiones que las normas 0 sus supervisores calificaban como perfectamente seguras resultaron ser muy riesgosas. Valores clasificados como AAA por las agencias de calificación crediticia, por ejemplo, causaron pérdidas significativas. Más recientemente, el banco franco-belga Dexia y bancos en Chipre tuvieron problemas por haber invertido en bonos del gobierno griego que habían sido considerados seguros por la normativa vigente. Los contratos de seguros de crédito emitidos por la compañía de seguros AIG antes de 2008, supuestamente utilizados para eliminar los riesgos, fueron honrados en su totalidad sólo porque AIG fue rescatada por el gobierno de los EE.UU.

Basilea III, que supuestamente incorporó normas más duras, todavía permite que los bancos puedan financiar hasta el $97 \%$ de sus activos con endeudamiento y así deja, tan sólo, un 3\% de capital para absorber pérdidas. Las propuestas de normas en EE.UU. llevarían este ratio de apalancamiento al $5 \%$ para los holdings de bancos y al $6 \%$ para las instituciones que reciben depósitos cubiertos por el seguro correspondiente. Otras exigencias se basan en complejos ponderadores de riesgo y en el uso de valuaciones que se apoyan sobre normas contables que a menudo no proporcionan una señal oportuna de dificultades financieras. Ningún análisis cuantitativo válido justifica este nivel de exigencias frente a las alternativas que requieren significativamente más capital.

Las ventajas de exigir mucho más capital propio son sustanciales. ${ }^{4}$ En primer lugar, con más capital los bancos serían capaces de absorber pérdidas mucho más grandes sin entrar en dificultades o volverse insolventes, como para necesitar rescates. Bancos más saludables son más confiables y, por lo tanto, un sistema en el que los bancos son más fuertes es menos frágil y más resistente. En segundo lugar, los bancos financiados con más capital toman mejores decisio-

\footnotetext{
${ }^{3}$ Las medidas de ratios de capital dependen de las normas de contabilidad y de las convenciones de cada país. Ver AH (2013a, Capítulo 6) y la Sección V de este trabajo.

${ }^{4}$ Para más detalles, ver ADHP (2013, Sección 2, 2014) y AH (2013a, Capítulo 6), que también analiza los enfoques normativos alternativos.
} 
nes de inversión, lo que los hace menos propensos a los conflictos de intereses discutidos anteriormente por ser simultáneamente prestatarios y prestamistas.

En tercer lugar, tener más capital reduce la intensidad con la que las instituciones financieras intenten reducir su apalancamiento en respuesta a las pérdidas (el llamado, desapalancamiento). Si un banco tiene sólo un 3\% de capital sobre activos, una pérdida del $1 \%$ en el valor de sus activos eliminaría un tercio de su patrimonio y podría llevar a la venta de una fracción grande de sus activos con el fin de pagar sus deudas y seguir invirtiendo. Cuando muchos bancos sufren pérdidas al mismo tiempo y están vendiendo activos similares con urgencia, las ventas pueden forzar a la baja los precios de los activos que poseen y debilitar aún más a los bancos. Con mucho más capital inicial, por ejemplo, el $20 \%$, una pérdida del $1 \%$ en el valor de los activos constituye sólo una pérdida del $5 \%$ del capital, y no daría lugar a ventas masivas de activos.

En cuarto lugar, un mayor capital reduce la probabilidad de que los bancos enfrenten problemas de liquidez. ${ }^{5}$ Los bancos son propensos a problemas de liquidez debido a que dependen de los depósitos y de otras deudas de corto plazo, que pueden ser retirados o generar necesidades de renovación frecuente, mientras que sus activos podrían no ser convertibles en efectivo de forma rápida y confiable, sobre todo en situaciones de estrés.

Los problemas de liquidez no son difíciles de abordar si se percibe que el prestatario es solvente. Los bancos centrales actúan como prestamistas de los bancos que enfrentan problemas de liquidez, pero los préstamos sólo son dados contra garantías que sean suficientemente sólidas. Aunque el seguro de los depósitos ha resuelto en gran medida el problema de las corridas ineficientes de los depositantes, los acreedores no asegurados pueden comenzar una corrida, y es más probable que esto ocurra si el banco enfrenta dificultades que pongan en duda su solvencia. ${ }^{6}$ Así, requisitos de capital más elevados, al reducir la probabilidad de que los bancos enfrenten dificultades financieras o se vuelvan insolventes, también reducen la probabilidad y la gravedad de los problemas de liquidez.

En quinto lugar, requisitos de capital más elevados reducen los subsidios de los contribuyentes asociados a las garantías públicas implícitas, que perversamente

${ }^{5}$ Ver AH (2013a, Capítulo 13). Gorton (2013, p. 5) argumenta que la principal función de los bancos es "producir deuda" con el fin de proporcionar liquidez, pero cuando los bancos asumen deuda (para proporcionar esa liquidez) y toman riesgos con el dinero prestado, alguien tendrá que absorber las pérdidas que pudieran ocurrir.

${ }^{6}$ Ver AH (2013a, Capítulo 3). 
fomentan la imprudencia y generan todavía más riesgo para los contribuyentes y para la economía en general. Las garantías de deuda, en particular las otorgadas a las instituciones consideradas demasiado grandes para quebrar, estimulan y recompensan el endeudamiento excesivo, la toma de riesgos, su crecimiento y una mayor complejidad. Exigir a los bancos un mayor fondeo con capital propio reduce su capacidad de beneficiarse de estos subsidios distorsivos y desproporcionados. Con una reducción de estos subsidios, las instituciones demasiado grandes podrían reducir su tamaño debido a la presión de los inversores, de forma similar a lo ocurrido en EE.UU. en los ochenta con la división de los conglomerados en instituciones menores.

Los acreedores que están asegurados o que creen que cobrarán sin importar lo que el banco haga aportan poco para controlar los riesgos que toman los bancos o dificultan el cierre de las instituciones insolventes. Así, los bancos tienen más probabilidades de persistir con dificultades financieras o en la insolvencia a menos que los supervisores interfieran. Requisitos de capital más elevados que obliguen a los bancos a retener beneficios y a captar nuevo capital cuando enfrentan dificultades financieras otorgaría transparencia a las entidades que son demasiado débiles. Como se vio en la crisis de las instituciones de ahorro y préstamo de los ochenta en EE.UU. o en la crisis japonesa, los bancos débiles o insolventes no realizan buenos préstamos nuevos y pueden llegar a ser imprudentes, lo que aumenta el daño a la economía. En este contexto, es perjudicial para los supervisores postergar una solución en lugar de intervenir inmediatamente. ${ }^{7}$

\section{Las fuerzas económicas decisivas}

Si tener más capital es tan beneficioso, ¿por qué los bancos optan por tener tan poco? ¿Habrá un costo para la sociedad por exigir significativamente más capital a los bancos y, en caso afirmativo, cuál sería la normativa "óptima"? Es útil comprender primero las fuerzas decisivas que juegan un papel en esta discusión.

Las empresas, en general, pueden crecer e invertir sin pedir prestado, usando sus propias ganancias y vendiendo acciones como fuentes de financiamiento de inversiones rentables. Por lo tanto, las empresas deben elegir su estructura de fondeo a través del tiempo. Una idea clave sobre las finanzas de empresas proviene del conocido análisis de Modigliani y Miller (1958). Esta idea, que se

${ }^{7}$ Ver ADHP (2013, Sección 9) y AH (2013a, Capítulos 4 y 11). 
enseña en cursos básicos de finanzas de todo el mundo y se explica en la mayoría de los libros básicos sobre finanzas corporativas, se basa en un principio básico de la conservación. ${ }^{8} \mathrm{Si}$ todos los riesgos asumidos en las inversiones realizadas quedan a cargo de quienes financian a la empresa, simplemente reasignar el riesgo entre dichos inversores no puede por sí solo cambiar el costo total de financiación de una empresa. Si la estructura de financiamiento importa, entonces, debe ser por razones distintas al hecho de que algunos valores sean más riesgosos que otros.

Esta observación es a veces expresada por medio de la afirmación de que bajo ciertos supuestos fuertes, el valor total de una empresa para sus inversores es independiente de su estructura de financiamiento. Sin embargo, como lo reconocen Modigliani y Miller (1963), los supuestos que dan lugar a esta "irrelevancia" de la estructura de fondeo no se sostienen en el mundo real. Por ejemplo, para todas las empresas y en muchos países, los pagos de intereses de la deuda pueden deducirse como gastos con fines impositivos. Por lo tanto, las empresas, en general, pagan menos impuestos al incrementar la participación de la deuda en su estructura de fondeo. Así, las normas tributarias penalizan el financiamiento con capital propio y sesgan a las empresas hacia el endeudamiento. No hay una buena justificación económica de esta preferencia impositiva, y desde hace mucho tiempo ha sido considerada como altamente distorsiva. La ventaja fiscal de la deuda es especialmente perversa para los bancos, debido a que premia y alimenta sus ya fuertes incentivos para endeudarse en exceso. ${ }^{9}$

A pesar de la ventaja fiscal de la deuda, la mayoría de las empresas no se endeudan fuertemente y algunas hasta toman prestado poco. Entre las principales razones de dicho comportamiento están los costos de una quiebra y los problemas de "agencia" asociados con las dificultades financieras y la insolvencia, discutidos en la sección anterior, que se reflejan en las condiciones de su endeudamiento al ser anticipados por los acreedores. ${ }^{10}$ La llamada teoría del equilibrio de la estructura de fondeo sugiere que las empresas aumentan la deuda hasta un nivel en el que la ventaja fiscal de la deuda es compensada por estos otros costos.

\footnotetext{
${ }^{8}$ Ver, por ejemplo, Berk y DeMarzo (2013).

9 Para una breve discusión y algunas referencias, ver ADHP (2013, Sección 4.1). Más recientemente Cochrane (2014) propone, por el contrario, utilizar los impuestos para desalentar el uso excesivo de deuda de corto plazo por parte de los bancos.

${ }_{10}$ Ver AH (2013a, Capítulo 9) y Berk y DeMarzo (2013). El valor total de una empresa muy endeudada también puede ser menor que el de una empresa con menos deuda y más capital debido al problema del sobreendeudamiento, el cual puede llevar a una subinversión en proyectos rentables.
} 
Este enfoque, y gran parte del análisis de financiamiento en la literatura académica, trata la decisión de fondeo como si ocurriera por única vez, al comienzo de la vida de la empresa. La estructura de financiamiento se elige para generar el máximo valor de la empresa para sus inversores, que en la etapa inicial es el mismo que el valor para sus propietarios iniciales. Una vez que el endeudamiento comienza, sin embargo, los directivos y los accionistas normalmente preservan una gran flexibilidad. Los contratos de deuda rara vez toman en cuenta las consecuencias de todas las decisiones futuras, y la posibilidad de comprometer por anticipado a los propietarios iniciales con decisiones específicas por un horizonte largo es limitada.

Las decisiones de inversión y de financiamiento realizadas en beneficio de los accionistas de una sociedad endeudada podrían no estar reflejando los intereses de todos los inversores de la empresa. La presencia de deuda influye en las decisiones de los accionistas o administradores, incluyendo las relacionadas con la evolución del apalancamiento de la empresa en el tiempo. Al igual que el problema de la subinversión que se señaló anteriormente (e identificado en Myers, 1977), el sobreendeudamiento crea una fuerte resistencia a la reducción del apalancamiento por parte de los accionistas, ya que el beneficio de dicha acción a menudo recae en su totalidad en los acreedores a costa de los accionistas. Es más, los accionistas pueden preferir aumentar el endeudamiento en forma excesiva para su beneficio a costa de los acreedores. ADHP (2014) exploran esta "asimetría del apalancamiento" (leverage ratchet effect), la cual crea una especie de adicción al endeudamiento por parte de agentes sobreendeudados.

La "asimetría del apalancamiento" y los otros conflictos de "agencia" son particularmente relevantes para los bancos. Sin embargo, las distorsiones no actúan para limitar el apalancamiento de los bancos, debido a que no se reflejan plenamente en el costo de endeudamiento de los mismos. Muchos de sus acreedores, como los depositantes, no especifican contratos que contemplen todas las acciones posteriores relacionadas. Garantías explícitas o implícitas, que son características de la banca, alimentan y permiten el tipo de adicción al endeudamiento y al riesgo que ya están presentes debido al alto nivel de apalancamiento. Las garantías convierten el conflicto deudor-acreedor estándar en un conflicto entre gerentes de los bancos y, por lo menos, algunos accionistas y los contribuyentes. Los problemas estándar de riesgo moral típicos de la actividad aseguradora agravan los problemas. 
Por lo tanto, el alto apalancamiento de los bancos puede explicarse fácilmente por las garantías que hacen que los bancos eviten el impacto de los costos de quiebra o condiciones más duras de crédito cuando toman más riesgos. Las garantías de fuente pública permiten a los bancos mantener su deuda en condiciones atractivas a pesar del alto nivel de apalancamiento y de los riesgos que toman. ${ }^{11}$ Una vez que la deuda ha sido tomada, los accionistas de los bancos consideran la reducción del apalancamiento y, por lo tanto, los requisitos de capital como costosos, ya que les obligan a asumir un riesgo que recae actualmente sobre los demás y a dejar de aprovechar las ventajas impositivas de la deuda. ${ }^{12} \mathrm{El}$ afán de los bancos por distribuir dividendos a los accionistas es otra manifestación de la "asimetría del apalancamiento" y del conflicto deudoracreedor. El dinero pagado a los accionistas ya no queda disponible para pagar a los acreedores. ${ }^{13}$

Los banqueros y los expertos en bancos afirman a menudo que las ideas de Modigliani y Miller no se aplican a los bancos. La batalla sobre la pregunta: "¿Modigliani y Miller se aplica a los bancos?" se remonta a décadas. Merton Miller dio la respuesta a esta pregunta en el abstract de un trabajo de 1995: "sí y no". Esta respuesta es válida para todas las empresas; es decir, "sí" para el concepto general y "no" con respecto a la "irrelevancia" de la estructura de fondeo, especialmente desde la perspectiva de los accionistas. ${ }^{14}$

El argumento de exigir mucho más capital a los bancos no se basa de ninguna manera en la presunción de que la estructura de fondeo es irrelevante para los bancos o para cualquier otro tipo de empresa. Más bien, se basa en la ponderación apropiada de los costos y beneficios para la sociedad de las diferentes estructuras de financiamiento de los bancos. Ninguno de los costos que mayores exigencias de capital generarían para banqueros o accionistas representaría un costo para la sociedad, ya que se basan exclusivamente en la transferencia de dichos costos a otros, y en la creación de daño colateral al incrementarse la fragilidad del sistema.

\footnotetext{
11 Ver ADHP (2014, Sección 4.2) y AH (2013a, Capítulo 9) para las discusiones de los efectos de garantías y subsidios.

${ }^{12}$ La asimetría del apalancamiento se explora en ADHP (2014). El análisis supone que no es posible para los accionistas renegociar los términos de la deuda con acreedores tan dispersos, un supuesto que es ciertamente verdadero para los bancos, cuyos acreedores incluyen a los depositantes.

${ }^{13}$ Brunnermeir y Oehmke (2013) muestran como la falta de capacidad para generar compromiso de cumplimiento crea una "carrera al acortamiento de los vencimientos" (rat race to maturity), donde los bancos acortan en repetidas ocasiones el vencimiento de su deuda como una forma de sacar provecho de los acreedores anteriores y de tranquilizar a los nuevos acreedores de que cobrarán. Ver también ADHP (2014) y AH (2013a, Capítulo 10).

${ }^{14}$ Para más detalles, ver AH (2013a, Capítulo 7 y 2014, puntos 3-6), y Pfleiderer (2010).
} 
El apalancamiento excesivo y dañino existente se debe y está estimulado por las garantías públicas subvaluadas y, aún más, por la ventaja fiscal del endeudamiento sobre el capital. Si los costos de financiamiento de los bancos aumentaran como resultado de requisitos de capital más altos, esto sólo corregiría las actuales distorsiones que llevan a los bancos, en respuesta a los incentivos presentes, a incrementar la inestabilidad del sistema financiero. Si el gobierno quiere subsidiar a las empresas o la adquisición de vivienda, debe encontrar formas mejores y más directas para entregar los subsidios que subvencionando a los bancos para que pidan prestado en exceso (en EE.UU. el endeudamiento para la compra de viviendas también es subsidiado a través de la posibilidad de deducir los pagos de intereses de los préstamos hipotecarios, lo que posiblemente aliente un endeudamiento hipotecario excesivo).

Los subsidios al fondeo con deuda otorgan a los bancos ventajas injustas, que las grandes instituciones son capaces de utilizar para numerosas actividades no bancarias, a menudo más atractivas que la concesión de préstamos tradicionales a empresas. Los subsidios también fomentan y permiten el crecimiento excesivo y la imprudencia. ${ }^{15} \mathrm{Al}$ mismo tiempo, las retribuciones de los administradores vinculadas al retorno sobre el capital incentivan el endeudamiento excesivo. ${ }^{16}$ El sistema inestable crea auges y caídas, y hace que la economía sea propensa a crisis costosas. En síntesis, tener bancos fondeados con muy poco capital es caro para la sociedad.

¿Quién se beneficia de un alto apalancamiento de los bancos? Los principales beneficiarios son los propios banqueros y las personas cuya fortuna está íntimamente atada a la suerte de los bancos. Los accionistas diversificados de los bancos, que también son contribuyentes y miembros de la sociedad, sufren la fragilidad del sistema, los préstamos ineficientes y los daños colaterales de las crisis financieras (incluyendo sus inversiones en otras sociedades). ${ }^{17}$

\footnotetext{
${ }^{15}$ Este punto está desarrollado en ADHP (2013, Sección 4), que responde a las críticas de Levitin (2014), las cuales señalan que no está claro que los beneficios superen los costos. Ver también la Sección VI, más adelante.

${ }^{16}$ Ver AH (2013a, Capítulo 8).

${ }^{17} \operatorname{Ver} \mathrm{AH}$ (2013a, Capítulo 8) y “The Great Bank Escape”, por Anat Admati, Project Syndicate, 31 de diciembre de 2012.
} 


\section{Falsas contraposiciones}

¿Por qué ha fracasado la regulación del capital? Una de las razones es que las fuerzas económicas fundamentales discutidas anteriormente parecen ser mal entendidas o ignoradas en el debate político y las decisiones terminan siendo influenciadas por posturas erradas. Aclarar estas cuestiones es esencial si se quiere mejorar la normativa.

ADHP (2013), cuya primera versión es de agosto de 2010, repasa una serie de afirmaciones que apoyan el "mantra" de que el "capital es costoso". Estas afirmaciones se clasifican en tres tipos: (i) las falacias - argumentos que son falsos o inconsistentes con principios económicos básicos-, (ii) los hechos irrelevantes -argumentos que confunden los costos privados y sociales-, y (iii) los mitos -argumentos basados en teorías inverosímiles y sin fundamento que ignoran muchas de las fuerzas que se discutieron anteriormente-.$^{18}$

Un conjunto de esas afirmaciones falsas sugiere que el cambio en la estructura de fondeo de los bancos los obligaría a reducir actividades socialmente valiosas. Una fuente particularmente insidiosa de la confusión se debe a la mala interpretación de las palabras que se utilizan. En la banca, pero no en otra parte, la palabra "capital" representa, en esencia, los fondos que no provienen de endeudamiento, como los aportes de los accionistas. Equivocadamente, se dice que los bancos "mantienen" o "dejan de lado" capital. ${ }^{19}$ El uso inusual de la palabra "capital", y las expresiones asociadas como las de "mantener" o "apartar" capital, conducen a la falsa impresión de que el capital del banco es una reserva de efectivo que no puede ser invertido. En realidad, los requerimientos de capital no restringen lo que los bancos pueden hacer con sus fondos. Esta confusión permite que afirmaciones falsas como "mayores requisitos de capital no permiten a los bancos hacer préstamos" se difundan y no sean desafiadas. ${ }^{20}$

Otro conjunto de afirmaciones falsas sugiere que requisitos de capital más elevados serían costosos si reducen el retorno sobre el capital de los bancos (ROE,

\footnotetext{
${ }^{18} \mathrm{AH}$ (2013a, b, 2014) utiliza la expresión "trajes nuevos de los banqueros" (banker's new clothes) —por analogía con el conocido cuento sobre la inexistente ropa de un emperador-para referirse a las afirmaciones erróneas. Muchas de ellas, pero no todas, se relacionan con la regulación del capital.

${ }^{19}$ Como ya se ha mencionado, algunos valores que no representan capital, como la deuda de largo plazo, y que, en principio, pueden utilizarse para la "absorción de pérdidas", se contabilizan como parte del "capital regulatorio". Sin embargo, en la crisis financiera dichos valores no absorbieron pérdidas pese a que los bancos recibieron rescates financieros y otros tipos de apoyo por parte de los gobiernos.

${ }^{20}$ Véase una lista (parcial) de las referencias en $\mathrm{AH}$ (2013a, Capítulo 1 y 6), y en AH (2014, puntos 1-2).
} 
por sus siglas en inglés). Sin embargo, como se explica en ADHP (2013, Sección 3.3) y en $\mathrm{AH}$ (2013a, Capítulo 8), los argumentos basados en el ROE están equivocados. ${ }^{21}$ No sólo esta afirmación no se sostiene cuando los bancos incurren en pérdidas, sino que contradice la idea básica de Modigliani y Miller (1958) discutida anteriormente (que el sólo hecho de que el capital sea más riesgoso que la deuda no hace que una estructura de financiamiento que tenga más capital sea más "costosa"). Asimismo, el establecimiento de objetivos de ROE para los gerentes de los bancos es peligroso. Los accionistas también pueden ser dañados por una exposición al riesgo excesiva generada por objetivos basados en el ROE.

Un supuesto generalizado pero falso es que el fondeo de los bancos con capital es fijo e inmutable. Se afirma que una mayor exigencia de capital obligaría a los bancos a reducir la captación de depósitos u otras formas de "creación de liquidez". ${ }^{22}$ Los modelos en la literatura sobre actividad bancaria comúnmente asumen que los bancos son propiedad plena de sus administradores y que no tienen capital externo. En realidad, por supuesto, la mayoría de los bancos son sociedades anónimas, y los más grandes tienen el mismo acceso a inversores de capital que cualquier otra empresa. Incluso los bancos de propiedad privada pueden aumentar su capital mediante la retención de ganancias. Sin embargo, la falta de capital y la fragilidad resultante de los bancos son a menudo tratados como si fueran inherentes o inevitables.

Los banqueros y los académicos especializados en bancos a menudo preguntan “¿de dónde vendría el capital adicional?" Ellos parecen creer que el nuevo fondeo con capital de los bancos requeriría de nuevos ahorros y de nuevas entradas de fondos en los mercados de capitales. Esta visión, sin embargo, se basa en un malentendido fundamental sobre los mercados de capitales. Si un banco emite más capital y utiliza los fondos obtenidos para comprar valores que cotizan en los mercados, los mercados de capitales se ajustarán a un nuevo equilibrio en el que los inversores que han vendido los otros valores mantendrán las acciones bancarias adicionales, ya que, como un fondo de inversión, la rentabilidad del banco en parte reflejará los rendimientos de esos

${ }^{21}$ Para un resumen del argumento, ver Anat Admati y Martin Hellwig, "The Case Against Banking's Case for Less Capital”, Bloomberg View, 4 de febrero de 2013. Ver también Anat Admati “Beware of Banks' Flawed Focus on Return on Equity", New York Times, 25 de julio de 2011. Cabe destacar que, como se señala en AH (2013a), tales afirmaciones falsas se expresaron también en los populares libros de texto de Fredric Mishkin, quien se desempeñó en posiciones de alto nivel en la Reserva Federal.

${ }^{22}$ Para ejemplos, ver ADHP (2013, Sección 3.2) y AH (2013a, Capítulo 6). 
otros valores. No se necesitan nuevos ahorros ni nuevas entradas de fondos en los mercados de capitales. ${ }^{23}$

Dado que los bancos son frágiles, muchos economistas que creen que el "libre mercado" crea resultados eficientes suponen que debe haber una "buena" razón para ello. Si el "libre mercado" crea resultados eficientes, entonces hay poca o ninguna distinción entre el costo privado y el costo social, y se trata de encontrar un modelo en el que comportamiento observado se demuestre como el resultado eficiente para luego afirmar que el modelo "explica" lo que vemos.

Sin embargo, como se discutió en la sección anterior, las decisiones de los bancos se pueden explicar fácilmente por sus propios incentivos y por la incapacidad de los mercados para producir resultados eficientes, a pesar de la ineficiencia y el daño que pueden causar a la sociedad. Tales explicaciones, y la distinción entre costos y beneficios privados y sociales, tienden a ser desechadas, ya que hacen hincapié en la necesidad de contrarrestar los incentivos distorsivos y corregir las distorsiones.

Entre las razones por las cuales los bancos son considerados especiales se encuentra la noción de que "crean dinero", lo que implica tomar deuda. La liquidez provista por los depósitos y otras formas de deuda de corto plazo es conveniente tanto para los depositantes como para otros acreedores. Sin embargo, este hecho por sí mismo no establece que los bancos deben estar altamente apalancados o que el alto nivel de apalancamiento que observamos sea socialmente eficiente. Si los retornos de las inversiones de los bancos son riesgosos, problemas de solvencia pueden hacer fácilmente que su deuda de corto plazo se vuelva ilíquida, porque en caso de quiebra la deuda se congela o porque, incluso antes de la quiebra, terceros no aceptarán obligaciones del banco como medio de pago o como garantía. ${ }^{24}$

Para evitar tal destrucción de beneficios de la liquidez, el banco necesita capital, pero los mecanismos que se discutieron anteriormente, en particular las distorsiones generadas por el alto nivel de apalancamiento existente, pueden hacer

${ }^{23}$ Ver ADHP (2013, Sección 7) para una discusión sobre el panorama general.

${ }^{24}$ El financiamiento únicamente con deuda podría funcionar en un mundo sin riesgos, como se supone en el modelo formal de DeAngelo y Stulz (2013). Su análisis también está viciado por el supuesto de que el beneficio de los consumidores relacionado con la liquidez de los depósitos puede ser apropiado por los bancos. Pero de acuerdo a los principios básicos de microeconomía, en los mercados competitivos los productores no pueden, en general, apropiarse de los beneficios del consumidor, excepto si el aumento de los costos marginales de la provisión de depósitos crea espacio para un "excedente del productor". 
que el nivel de fondeo con capital sea socialmente ineficiente. Esta observación es a menudo ignorada en las publicaciones académicas referidas a los bancos, donde la mayoría de los modelos que se centran en la creación de liquidez asumen que el capital es escaso o no está disponible para los bancos. ${ }^{25}$ Cuando los problemas de liquidez amenazan la solvencia de los bancos, los argumentos basados en la provisión de liquidez se utilizan para justificar la asistencia y los rescates de los bancos centrales.

Algunas investigaciones académicas afirman que la deuda de corto plazo resuelve el conflicto de intereses entre administradores e inversores aplicando "disciplina" a los administradores de los bancos. Por ejemplo, en un libro de destacados académicos que se propone orientar la política económica, los autores afirman como una cuestión de hecho del mundo real: "Los requisitos de capital no son gratuitos. El efecto disciplinador de la deuda de corto plazo, por ejemplo, hace que la administración sea más productiva". ${ }^{26}$ Estas afirmaciones se basan en modelos teóricos altamente inverosímiles que carecen de evidencia empírica válida y relevante. ${ }^{27}$

De hecho, los depositantes y los acreedores de corto plazo no pueden proporcionar "disciplina" si están asegurados y si tienen pocos incentivos o capacidad para recopilar información, y si los administradores pueden seguir endeudándose continuamente. El efecto de "asimetría del apalancamiento" y la "carrera al acortamiento de los vencimientos", que representan lo contrario a la disciplina, explican completamente el comportamiento de los bancos. ${ }^{28}$ El hecho de que existan las corridas contra los bancos no prueba la teoría de la disciplina así

\footnotetext{
${ }^{25}$ En los últimos años, los fondos de money market y los mercados de deuda de corto plazo, tales como los mercados de pases (en donde el endeudamiento implica vender un activo y la promesa de recomprarlo a futuro), han crecido de forma espectacular. AH (2013a, Capítulo 10) discute los temas relacionados con la provisión de dinero y de liquidez por parte de los bancos, y sostiene que la provisión de liquidez se ve perjudicada por el alto nivel de apalancamiento, y que se vería favorecida por los requisitos de capital más elevados. Ver también, ADHP (2013, Sección 7) y AH (2014, puntos 5-6).

${ }^{26}$ Ver (French et al., 2010, p. 69). En "Love the Bank, Hate the Banker", de Raghuram Rajan, Project Syndicate, 27 de marzo 2013, se afirma que "la necesidad de pagar o refinanciar la deuda impone disciplina, dando al banquero un mayor incentivo para gestionar el riesgo con cuidado". Sin embargo, no se explica cómo se logra esta supuesta disciplina.

${ }^{27}$ Por lo que se relata en $\mathrm{AH}$ (2013b), un veterano de 30 años de la industria bancaria, tras la lectura de la larga sección dedicada a la "disciplina de la deuda" en una versión anterior de ADHP (2013), preguntó por qué se había escrito tanto sobre ese tema, diciendo: “¿es esto algo académico?” Ver AH (2013b) y ADHP (2013, Sección 5) para una amplia discusión. Pfleiderer (2014) también analiza la inverosimilitud de estos modelos y su mal uso.

${ }^{28}$ Ver AH (2013b) y ADHP (2013, Sección 5) para una discusión detallada. Brunnermeier y Oehmke (2013) muestran cómo una "carrera al acortamiento de los vencimientos" puede deberse a las dificultades para obligarse a no dañar a los acreedores anteriores.
} 
como el hecho de que mucha gente fume no prueba que el fumar sea bueno para la salud. Tal teoría no explica por qué se fuma.

Curiosamente, los argumentos de liquidez y "disciplina" relacionados con la deuda de los bancos muestran una incompatibilidad de lo que se afirma acerca de los roles de los depositantes y de los acreedores de corto plazo. En el argumento de la liquidez, los depositantes no monitorean a los banqueros; sólo quieren que la deuda del banco sea segura y líquida. Aquellos que adhieren a esta visión perciben al seguro sobre los depósitos y a las garantías como útiles para la estabilidad financiera. El argumento de la "disciplina", por el contrario, exalta las virtudes de la fragilidad, suponiendo que los depositantes gastan tiempo y dinero para controlar a los administradores de los bancos. Los seguros de los depósitos, establecidos debido al enorme costo para la sociedad de las corridas que se requieren para generar la "disciplina", eliminan los incentivos a reunir información sobre los bancos y contrarrestan el realismo del argumento. ¿Se deberían anular, entonces, los seguros de los depósitos para resolver el problema de gobernanza en los bancos, el cual es resuelto de otra manera por otros tipos de empresas? ${ }^{29}$

La afirmación de que "el capital es costoso" es justificada a veces en la literatura bancaria haciendo alusión a la "información asimétrica", y citando a Myers y Majluf (1984). ${ }^{30}$ El argumento de Myers-Majluf, sin embargo, se limita a las nuevas emisiones de acciones comunes, y no se aplica a las nuevas acciones emitidas a los accionistas existentes, ni puede explicar la resistencia a retener ganancias. Myers y Majluf (1984), en realidad hacen hincapié en que con las asimetrías de información que consideran, la obtención de fondos mediante la retención de ganancias es preferible al nuevo endeudamiento. Y su artículo es considerado la base de la denominada teoría del orden de preferencia del financiamiento (pecking order theory), donde los resultados no distribuidos son la fuente de financiación preferida. Más aún, cuando las reducciones de apalancamiento son impuestas por la regulación, la selección adversa se vuelve irrelevante. Cualquier "costo de dilución" para los accionistas de las empresas con perspectivas de retorno superior a la media se igualarían con los benefi-

\footnotetext{
${ }^{29}$ Gorton (2012) alude al "periodo de calma" provocado por el seguro de los depósitos y cree que las garantías protegen la valiosa liquidez de la deuda de los bancos. Por el contrario, Diamond y Rajan (2012) señalan en el abstract de su trabajo como un hecho del mundo real: "Los bancos financian activos ilíquidos con depósitos de exigibilidad inmediata, lo que disciplina a los banqueros pero los expone a corridas dañinas", y sugieren que el seguro de los depósitos interfiere con este beneficio de tener depósitos.

${ }^{30}$ Tal afirmación se hace, por ejemplo, en Bolton y Freixas (2006) y Calomiris (2013).
} 
cios de los accionistas de las empresas con perspectivas de retorno inferiores a la media. ${ }^{31}$

En resumen, gran parte de la literatura académica ha aceptado la fragilidad de los bancos como inherente a su funcionamiento o inevitable, y algunos hasta han tratado de "explicar" su utilidad. Sin embargo, antes de utilizar un modelo teórico para avalar decisiones públicas, es esencial asegurarse de que sea relevante, sobre todo si hace caso omiso de las posibles fuerzas económicas discutidas en la Sección III. Las distorsiones y problemas de "agencia" creados por el alto apalancamiento no desaparecen en el caso de los bancos, de hecho, a menudo son fundamentales para la comprensión de las distorsiones en el sector bancario y, por lo tanto, para tratar de corregirlas. Sin embargo, frecuentemente son dejadas de lado en favor de otras, y más convenientes, "fricciones". La cultura académica también trata a todos o a la mayoría de los modelos como "relevantes hasta que sean rechazados por pruebas empíricas definitivas", aun cuando algunos modelos pueden ser rechazados mediante simples filtros basados en todo lo demás que sabemos. Estos puntos y la posible mala utilización de los modelos teóricos se discuten en detalle en Pfleiderer (2014).

Los grupos de presión bancarios amenazan con el argumento de que requisitos de capital más elevados reducirán los préstamos. Pero ninguna teoría o estudio empírico ha demostrado que los préstamos se reducirían si se les solicitara a los bancos retener ganancias o emitir nuevas acciones cuando sus niveles de capital se reducen por pérdidas. Hay pruebas significativas de que los bancos mejor capitalizados sostienen el nivel de préstamos en tiempos de crisis, y que lo que perjudica el otorgamiento de préstamos es la insuficiencia de capital y no que se exija "demasiado". ${ }^{32}$ Los auges de crédito generalmente implican préstamos excesivos y dispendiosos, sólo para ser seguidos por crisis que luego restringen los préstamos a los deudores valiosos.

\footnotetext{
${ }^{31}$ Para una discusión detallada, ver ADHP (2013, Sección 6) y ADHP (2014, especialmente la Sección 4.2.5), que muestran que la resistencia a la reducción del apalancamiento es un fenómeno generalizado y que la información asimétrica no proporciona una explicación completa del comportamiento observado de los bancos.

32 Ver ADHP (2013, Sección 9) y Cole (2013).
} 


\section{Hacia una mejor regulación de apalancamiento}

Los niveles de capital de los bancos se han reducido en los últimos 150 años. Esta disminución está relacionada, al menos en parte, con la expansión de las redes de seguridad de los bancos, en la forma de asistencia del banco central, seguro de los depósitos y garantías implícitas. Al mismo tiempo, la naturaleza y el alcance de los riesgos que los bancos toman, la naturaleza de sus deudas (sin considerar a los depósitos), el entorno legal y regulatorio y el alcance global de los bancos, también han evolucionado, lo que dificulta las comparaciones históricas. ${ }^{33}$ Las fuerzas económicas discutidas en la Sección III, sin embargo, sugieren que aun si los bancos han sido siempre frágiles, no se sigue que su nivel de fragilidad sea inevitable, esencial o eficiente. El statu quo actual, que refleja las decisiones de los bancos y una regulación ineficaz, por lo tanto, no es una referencia útil para lo que la regulación debe tratar de lograr.

Es evidente que las normas en vigor antes de 2008 no lograron controlar el apalancamiento de manera efectiva. Martin Wolf, editor de economía del Financial Times, dijo acertadamente en 2010 refiriéndose a las reformas de Basilea III: "Triplicar los requerimientos previos parece duro hasta que uno se da cuenta de que triplicando casi nada no resulta en mucho". ${ }^{34}$

Pero, ¿cuánto capital debe exigirse? El espíritu de la respuesta es capturado por John Cochrane: "ilo suficiente como para que ya no importe!" Lo suficiente para que nunca más oigamos el pedido de que "ilos bancos deben recapitalizarse!" (a expensas del contribuyente)". ${ }^{35} \mathrm{AH}$ (2013a) proponen que el requisito

${ }^{33}$ Ver AH (2013a, Capítulo 2) y referencias (sobre todo en las notas 20-27, pp. 242-243). Calomiris (2013) y Levitin (2014) discrepan con las cifras históricas que se presentan en esos trabajos, pero las referencias citadas sostienen el punto. Más importante aún, la propuesta de ratios de apalancamiento no descansa en ninguno de estos datos históricos, sino que se basa más bien en argumentos económicos, en los datos de apalancamiento de otras industrias no reguladas y en el costo social del apalancamiento de los bancos. Como se argumenta en AH (2013a, 2014), la economía de los mercados de acciones y de alto nivel de apalancamiento no son fundamentalmente diferentes para los bancos, incluso si parte de la deuda de los bancos es útil para proporcionar liquidez. Levitin (2014) también señala que el mercado no demanda ratios de capital de $20 \%$ o $30 \%$ a los pequeños bancos que pueden quebrar o a los bancos de inversión. Pero los bancos pequeños tienen depósitos asegurados, que no soportan los costos de quiebra, y los acreedores de Lehman Brothers bien pueden haber pensado que cobrarían en su totalidad, al igual que los acreedores de Bear Stearns, a pesar de que no estaban asegurados de manera explícita. Es evidente que la quiebra de Lehman Brothers tuvo importantes daños colaterales. Como se explica en ADHP $(2013,2014)$, los mercados pueden permitir que el apalancamiento sea ineficientemente alto, tanto desde el punto de vista social como del privado. Exigir a los bancos de inversión, que pueden expandir el riesgo y convertirse en sistémicos, tener ratios de capital del $30 \%$ tiene beneficios sociales con un costo relevante mínimo.

${ }^{34} \mathrm{Ver}$ "Basel III, the Mouse that did not Roar", por Martin Wolf, Financial Times, 13 de septiembre de 2010.

${ }^{35}$ Ver "Running on Empty", de John Cochrane, Wall Street Journal, 1ro. de marzo de 2013, disponible en http://johnhcochrane.blogspot.com/2013/03/the-bankers-new-clothes-review.html. 
de capital sea fijado en el $30 \%$ de los activos totales, con una ajuste gradual regulatorio si el requisito no se cumple. La idea es que cuando el ratio de capital sea inferior al 30\%, el banco no pueda distribuir dividendos entre los accionistas, y si está por debajo del $20 \%$ estaría obligado a recapitalizarse con nuevas emisiones de acciones.

Estos números aproximados no deben tomarse literalmente, sólo sugieren que los niveles de capital deben ser mucho mayores que los ratios sugeridos en las propuestas actuales. La mención de números concretos, tiene el único objetivo de subrayar que se requiere una reforma radical de la regulación del capital, no un retoque. En la aplicación de la regulación, el diablo y el desafío están claramente en los detalles.

De hecho, la especificación de tales ratios no tiene sentido sin la discusión de cómo se determinan el numerador y el denominador. Por ejemplo, AH (2013a, Capítulo 6) muestran que la relación entre el capital y los activos totales de JPMorgan Chase a finales de 2011 fue de aproximadamente el $8 \%$ si los activos se medían de acuerdo con las normas contables de Estados Unidos, pero sólo del $4,5 \%$ con las normas contables utilizadas en la mayoría de los países europeos. La diferencia está relacionada con el tratamiento de los derivados.

La forma en la que los reguladores tratan a los activos que la contabilidad permite dejar fuera del balance es de suma relevancia, así como las numerosas maneras en las que se pueden valuar los activos y pasivos de los bancos. $\mathrm{Si}$, por ejemplo, los pasivos pueden valuarse "a precios de mercado", una empresa en dificultades cuya deuda se vuelve menos valiosa puede de repente registrar un mayor nivel de patrimonio neto, a pesar de que sus compromisos de deuda actuales están determinados por los pagos prometidos de la deuda y no por su valor de mercado.

Al mismo tiempo, la contabilidad basada a valores de libro, que a menudo dependen de transacciones históricas, tienden a ajustarse lentamente a la nueva información, sobre todo en el caso de las pérdidas de las carteras crediticias de los bancos. Haldane (2011a) muestra que los valores de libro no distinguieron entre bancos débiles y fuertes durante la crisis financiera, mientras que los valores de mercado proporcionaron una información más útil y oportuna. Al reconocer este problema, AH (2013a, p. 190) proponen: "Los supervisores deberían considerar otras fuentes de información [que los valores de libros], tales como 
los precios de las acciones y otros indicadores de mercado, en el intento de mantener la seguridad y solidez del sistema financiero. Cualquier preocupación acerca de la acumulación de riesgos debería dar lugar a medidas prudentes, tales como la prohibición de los pagos de dividendos a los accionistas, para evitar el agotamiento del capital". ${ }^{36}$

Por desgracia, no parece haber un modelo matemático bien calibrado ni datos confiables y relevantes para establecer con precisión cuál debería ser el ratio de requisitos de capital, especialmente teniendo en cuenta las cuestiones de medición. Pero, de nuevo, no se sigue que debamos permanecer en un statu quo peligroso. Las regulaciones de capital actuales y propuestas son también arbitrarias, y niveles más elevados son fáciles de justificar, incluso si no son precisos, sobre la base de las cuestiones discutidas en la Sección III y de la capacidad de los bancos para utilizar sus ganancias y para acceder a los mismos mercados que otras empresas usan de forma rutinaria con el fin de aumentar su capital.

La pregunta de por qué los requerimientos deberían ser del $30 \%$ se puede dar vuelta para preguntar: ¿por qué no? Habiendo formulado esta pregunta por más de cinco años, no he logrado recibir una respuesta válida. Después de todo, sin regulación, las empresas saludables a menudo usan sus ganancias para financiar inversiones. Los accionistas tienen derecho a los beneficios siempre y cuando se paguen las deudas, y los precios de las acciones reflejan las expectativas de los inversores sobre estos beneficios futuros. El hecho de que, con niveles de capital por debajo del $10 \%$ de sus activos, los bancos rutinariamente distribuyan dividendos a sus accionistas y agoten su capital refleja sus incentivos para permanecer altamente apalancados, pero no habría ningún costo social si se los obligara a utilizar sus ganancias para reducir deuda o para invertir en nombre de sus accionistas, al tiempo que haría más segura su deuda. ${ }^{37}$

\footnotetext{
${ }^{36}$ Calomiris (2013) critica AH (2013a) por hacer foco en el patrimonio neto contable y no en lo que él llama patrimonio "actual" o "verdadero". Esta crítica no tiene en cuenta lo expresado en esta parte del trabajo, que reconoce claramente la dificultad de utilizar los valores contables para la regulación, ni la naturaleza aproximada de las sugerencias que se realizan sobre el nivel de los ratios de capital. Las recomendaciones sobre la manera de implementar la regulación se basan en el uso de capital "verdadero" para absorber pérdidas, a través de utilidades retenidas y de la emisión de nuevas acciones. En AH (2013a) no se especificaron los detalles técnicos debido a que apunta al público general, pero estos puntos de vista son compartidos por muchos académicos prominentes; ver, por ejemplo, "Healthy Banking System is the Goal, Not Profitable Banks", Financial Times, 9 de noviembre de 2010 (texto disponible en http://www.gsb.stanford.edu/news/research/admatiopen.html).

${ }^{37}$ Para una discusión general del tema, ver ADHP (2013, Sección 7). Un modelo de la economía que pudiera captar esta visión podría proporcionar una justificación analítica para los requisitos de capital más elevados.
} 
A los bancos cuyas acciones cotizan en la bolsa de valores podría solicitárseles que emitan una determinada cantidad de nuevo capital. Si un banco no puede aumentar su capital a ningún precio, hay razones de peso para creer que puede ser insolvente, demasiado opaco o demasiado débil para sobrevivir sin subsidios. Esta "prueba de resistencia" basada en el mercado sería más informativa que aquellas realizadas por los bancos y sus supervisores. Los bancos insolventes son disfuncionales y perjudiciales; y no deberían persistir.

Otra falla en la normativa de capital actual es el intento de calibrar los requisitos utilizando un sistema de ponderadores de riesgo altamente complejo y manipulable. Como se discute en AH (2013a, Capítulo 11), este enfoque se basa en la ilusión de una ciencia exacta, pero ignora riesgos importantes y ha exacerbado la fragilidad del sistema. También crea distorsiones y sesgos que tienden a desalentar los préstamos tradicionales en favor de otras inversiones. Si los requisitos de capital fueran mucho más altos en relación con el total de activos, los bancos tendrían mayores incentivos para medir y gestionar el riesgo de manera más eficaz, y el seguimiento detallado del supervisor sería menos importante.

Además, la normativa vigente permite, y muchos lo han defendido, que los títulos de deuda que se convierten en capital en algunos escenarios, como los bonos contingentes convertibles en capital, sean contabilizados como capital regulatorio. ${ }^{38}$ Como se discute en AH (2013a, Capítulo 11) y ADHP (2013, Sección $8)$, estos son sustitutos problemáticos y no probados. Aunque parezcan reducir los costos de quiebra, es interesante observar que dichos valores no son tan utilizados por entidades no bancarias para sustituir capital.

Otros enfoques de la normativa bancaria se basan en los mecanismos de reestructuración que esencialmente permiten a los bancos incumplir una parte de sus deudas, imponiendo quitas a algunos acreedores. Estos enfoques plantean muchas dudas, porque en el momento en el que el proceso de reestructuración se dispara, los mercados pueden ya estar siendo afectados por la incertidumbre sobre el comienzo y el funcionamiento de dicho proceso. Exigir más capital tiene por objeto prevenir el estrés financiero y el incumplimiento. Cuando se producen pérdidas, alguien debe soportarlas, y los accionistas que se benefician de los buenos tiempos son los candidatos más adecuados. Debido a que no existe un compromiso legal para pagar a los accionistas, estos soportan las pérdidas de

${ }^{38}$ Algunos continúan defendiendo los valores híbridos con diferentes variedades de características de diseño. Ver, por ejemplo, Calomiris (2013). 
forma automática, sin la necesidad de complejos y posiblemente largos y costosos procesos legales. ${ }^{39}$

Una pregunta legítima es: ¿por qué no exigir requisitos de capital aún más elevados? El reciente premio Nobel Eugene Fama sugirió en 2010 que una exigencia de capital del $50 \%$ sería apropiada. Para aquellas instituciones que pueden fácilmente expandir su riesgo y que están altamente conectadas con otras instituciones financieras, mayores requisitos podrían ser de hecho deseables. ${ }^{40}$ Tales niveles de capital eran comunes en el siglo XIX, cuando la mayoría de los bancos eran sociedades comerciales privadas con responsabilidad ilimitada del propietario y no había una red de seguridad de los bancos centrales o los gobiernos (ver Haldane, 2011b).

Los bancos ya no presentan dichas formas de control de la propiedad, en tanto, la mayoría tiene acceso a los mercados de capitales, donde los inversores pueden compartir su riesgo. Aumentar la fracción de capital de responsabilidad limitada es similar a incrementar la responsabilidad total de la institución. Si las instituciones más grandes se reducen como resultado de tener que utilizar más capital porque ya no son capaces de depender del fondeo con deuda subsidiada, un menor tamaño será probablemente más apropiado y eficiente para la economía (y también para los que controlan la institución).

Recientemente, han ido aumentando las voces a favor de la "banca restringida" (narrow banking), donde los depósitos están respaldados por una reserva en efectivo del $100 \%$ (o de títulos públicos). Algunos también piden que toda la intermediación financiera dependa totalmente del financiamiento con capital, anulando así las funciones de liquidez y transformación de plazos que cumplen los bancos. ${ }^{41}$ Sin embargo, es poco probable que las fricciones de información que han generado dichas funciones eliminen del todo la fragilidad, y pueden ser tan difíciles de administrar como las propuestas menos extremas que requieren mucho más capital pero que siguen permitiendo que los pasivos de

\footnotetext{
${ }^{39}$ Por supuesto, la idea de los bonos contingentes convertibles y de los procesos de reestructuración es tratar de hacer que las pérdidas para los acreedores sean de alguna manera automáticas. Pero esto significa que los contratos de deuda tienen que especificar con precisión cómo se determinarán dichas pérdidas. Invariablemente, dada la complejidad de los pasivos de los bancos y la opacidad de sus estructuras, esta especificación y la forma en la que podría implementarse, sobre todo en una situación de crisis, plantean cuestiones no triviales de control sobre el momento en el que el disparador del proceso puede activarse.

${ }^{40}$ Entrevista CNBC, mayo de 2010.

${ }^{41}$ Ver, por ejemplo, Cochrane (2014), y Martin Wolf, "Strip Private Banks of their Power to Create Money", Financial Times, 24 de abril de 2014.
} 
los bancos sean más líquidos y sujetos a menos fricciones de información que sus activos. ${ }^{42}$

El hecho de obligar a los depositantes asegurados a colocarse en un "banco restringido", lo cual reduce el fuerte efecto de "asimetría del apalancamiento" que crean los primeros cuando se los incluye en la estructura de financiamiento de instituciones tomadoras de riesgo, es una característica atractiva de este tipo de banca. Pero la "banca restringida" probablemente constituiría una parte muy pequeña del sistema financiero, como se vio en la migración de depósitos hacia los fondos de money market que pagaban tasas de interés más elevadas. A menos que sean regulados de manera efectiva, las instituciones no autorizadas a tomar depósitos pueden convertirse en objeto de corridas y ser perjudiciales para el sistema al quebrar. Las consecuencias del colapso de Lehman Brothers, que era un banco de inversión, son un claro ejemplo de ello.

El hecho de no utilizar deuda para la intermediación financiera no eliminaría necesariamente la fragilidad y los posibles daños a los pequeños inversores. Los inversores quieren que la mayor parte de su dinero gane intereses y que a la vez sea líquido para ser utilizado con cierta certeza cuando lo necesiten. Si los bancos deben pasar a operar como fondos comunes de inversión sin deuda, los inversores que necesiten efectivo venderán sus cuotas partes y obtendrán el valor que las mismas registren en el momento. Determinar el valor de dichas cuotas partes sería sencillo si los activos del fondo (o el fondo mismo) se transaran diariamente en mercados de valores, pero en caso contrario, los fondos de inversión también podrían sufrir algo parecido a corridas si los cuotapartistas temen bajas importantes en los precios de los activos, venden sus participaciones y el fondo tiene que liquidar apresuradamente sus activos. ${ }^{43}$

Operar en los mercados bursátiles expone a los individuos que necesitan vender por razones de liquidez a pérdidas con respecto a los inversores mejor informados. La opacidad de los activos como hipotecas y otros préstamos generan incentivos para que aquellos que tienen un mejor acceso a la información participen en dicha operatoria si las acciones de los bancos con $100 \%$ de capital

${ }^{42}$ La experiencia de la crisis sugiere que si los fondos de money market son parte del sistema de pagos y compiten con los depósitos, deberían ser tratados como los bancos y regulados de manera acorde.

${ }^{43}$ Gordon y Gandía (2013), por ejemplo, muestran que los fondos de money market con un valor flotante también fueron bastante inestables, al mismo tiempo que los fondos que aseguraban un valor neto fijo estaban experimentando corridas en 2008. Debido a que Alemania había tenido experiencias similares con fondos comunes "abiertos" para inversiones inmobiliarias, el Ministerio de Hacienda propuso en julio de 2012 prohibir la operatoria de fondos comunes para dicho tipo de inversiones. 
se negociaran en los mercados de valores. La insensibilidad de la deuda de los bancos a la información es valiosa para la provisión de liquidez, y la idea de exigir ratios significativos de capital (30\% o más), pero no el 100\%, intenta preservar dicha función y lograr un equilibrio entre la provisión de liquidez y la estabilidad del sistema financiero.

Los requisitos mínimos de capital son sólo el primero de los tres "pilares" del marco regulatorio de Basilea. Los otros incluyen recomendaciones para la supervisión y para una mayor transparencia. Antes de la crisis financiera, los supervisores no cuestionaron las numerosas prácticas imprudentes de los bancos. Este fracaso ha desempeñado un papel importante para la acumulación de riesgo en el sistema. Como AH (2013a, p. 190) señalan, "los supervisores deben tener en cuenta que su tarea principal es proteger al público. Las preocupaciones acerca de los detalles de los requisitos regulatorios, las normas de contabilidad y otras medidas no deben desviar la atención de este objetivo".

En la discusión sobre la regulación bancaria, políticos, reguladores e incluso académicos a menudo parecen preocupados con la competitividad de "sus" bancos nacionales. Estas preocupaciones están fuera de lugar y no deben interferir con la necesidad fundamental de mantener la estabilidad financiera. Como se ha visto recientemente en Irlanda, Islandia y Chipre, los bancos "exitosos" pueden causar un gran daño a la economía. Permitir bancos imprudentes que ponen en peligro al público, sólo porque otros países tontamente permiten a sus bancos hacerlo, es una mala política. ${ }^{44}$

Una preocupación relacionada es que una regulación más estricta podría llevar a determinadas actividades a migrar al poco regulado o, directamente, no regulado "shadow banking" (sistema bancario paralelo) de los fondos de money market, los hedge funds y otras instituciones. Esta preocupación, sin embargo, sólo confirma la importancia de lograr una aplicación efectiva de la normativa, cuestión con la que se debe lidiar en cualquier caso.

De hecho, el surgimiento del shadow banking, que de hecho interactúa con las instituciones reguladas y cuyas entidades son visibles para los reguladores, es

\footnotetext{
${ }^{44}$ Ver, por ejemplo, French et al. (2010) y Levitin (2014). AH (2013, Capítulo 12) demuestran por qué esos argumentos no son válidos. Aunque la interconexión puede conducir a que el fracaso de una institución en un país afecte a las instituciones en otras partes, son principalmente los contribuyentes nacionales los que pagan los recates de las instituciones reguladas en sus países. Para evitar una "carrera hacia el fondo" en la regulación, cada regulador debería concentrarse en lo que pueda hacer por la estabilidad financiera global que afecta a los ciudadanos de su país.
} 
una clara evidencia de las fallas en la aplicación de la regulación previa. ${ }^{45}$ Los reguladores no pudieron identificar la significativa exposición de las instituciones reguladas a entidades como los "vehículos especiales" (special purpose vehicles) o los fondos de money market. Los riesgos asumidos por las entidades no reguladas se materializaron y afectaron a todas las instituciones justo cuando todo el sistema ya estaba débil. En lugar de renunciar a la regulación esencial y sufrir las consecuencias, debe afrontarse el siempre presente desafío de diseñar y hacer cumplir las regulaciones. Es posible hacerlo.

\section{El desafío político}

Más allá de las confusiones o los malos entendidos, una razón clave del fracaso reiterado para aplicar una regulación efectiva es la economía política de la banca. Los bancos son tan frágiles porque quieren y porque los hacedores de política económica suelen verse beneficiados (o por otras causas) por tolerar e, incluso a veces, alentar esta fragilidad, y tienen poco para ganar si la desafían. La ceguera voluntaria a los daños causados por un sistema frágil ayuda a los banqueros y a los hacedores de política económica a ignorar y a pasar por alto los riesgos, y también a defenderse de las críticas.

Todos los sectores presionan por subsidios, pero los subsidios otorgados a través de garantías implícitas son especialmente fáciles de proveer para los hacedores de política, ya que no aparecen en los presupuestos y parecen invisibles o gratuitos. Las amenazas de la industria de que las regulaciones duras son perjudiciales, el miedo de los políticos a que los bancos no financien sus causas, los argumentos de que las crisis financieras son similares a los desastres naturales y que los esfuerzos de reforma están en camino tapan los fracasos de todos los involucrados. ${ }^{46} \mathrm{~A}$ pesar de la indignación pública por la crisis financiera y del enorme daño que causó, los temas y los detalles de la regulación no son ampliamente conocidos. Esta situación permite que las políticas equivocadas persistan.

\footnotetext{
${ }^{45}$ Como se discutió en $\mathrm{AH}$ (2013a, especialmente en el Capítulo 10 y pp. 224-226) el problema de hacer cumplir la regulación es importante, y si bien surgió con las regulaciones anteriores debe abordarse de todos modos.

${ }^{46} \mathrm{AH}(2011,2013$, Capítulo 12) discuten la necesidad de centrarse en la estabilidad financiera y en el daño que puede ser causado por el conflicto de objetivos políticos. Ver también "Foreign regulators say U.S. OCC should improve bank oversight", Reuters, 5 de diciembre de 2013, que critica a la Oficina del Contralor de la Moneda por preocuparse por la competitividad de los bancos de Estados Unidos en lugar de centrarse principalmente en el control del riesgo para el público. Levitin (2014) proporciona un análisis útil del marco regulatorio en los EE.UU. y del problema de la captura del regulador.
} 
Un ejemplo de la "economía política de las posturas erróneas", fue el debate a mediados de la década de 1990 con respecto a los cambios en las normas contables de Estados Unidos para exigir que el otorgamiento de opciones sobre acciones a ejecutivos fuera considerado un gasto a deducir de las ganancias. Muchas de las posturas erróneas que pueden encontrarse en el debate del apalancamiento de los bancos — falacias, hechos irrelevantes y mitos-fueron respaldadas. Afirmaciones sin fundamento, del tipo "la innovación se verá perjudicada", funcionaron, haciendo que los cambios propuestos en las normas contables no se llevaran a cabo. Una década más tarde, después de los escándalos contables de Enron, WorldCom y otros, el cambio se realizó y ninguna de las predicciones hechas por los grupos de presión se materializó. ${ }^{47}$

Las relaciones simbióticas entre los bancos y los gobiernos, y la falta de suficiente participación pública, son un obstáculo para la reforma. Esta situación y la observación de que muchos de los involucrados en el debate, o bien no entienden completamente los problemas o tienen razones para sostener los argumentos erróneos, ha llevado a Martin Hellwig y a la autora del presente trabajo a tratar de explicar los problemas de forma más accesible para un público más amplio. Para que los hacedores de política se dediquen a los aspectos más técnicos de cómo diseñar e implementar las regulaciones, primero deben enfrentar estos temas. En este sentido, la experiencia de ambos de intentar participar en el debate de políticas desde el año 2010 ha sido decepcionante.

En el prefacio de la edición de bolsillo de AH (2013a), se declara: "hemos escrito este libro para informar y estimular a más personas a participar en el debate. Al explicar los temas en un lenguaje sencillo, hemos querido crear un grupo más amplio para una reforma financiera eficaz. La ampliación de este grupo de participación es esencial para lograr un cambio". El esfuerzo continuará con la esperanza de que, a pesar del desafío político que implica, puedan obtenerse resultados en el largo plazo. ${ }^{48}$

\footnotetext{
${ }^{47}$ Para una discusión extendida y algunas referencias, ver los comentarios finales de ADHP (2013).

${ }^{48}$ La economía política de la banca y la forma en la que regulación está organizada son diferentes en los distintos países, pero los incentivos para elegir el apalancamiento excesivo son generalizados. Incluso sin el problema de la captura, la regulación puede fallar si los costos y beneficios pertinentes son mal interpretados. AH (2013a) y los libros de Sheila Bair, Jeff Connaughton y Neil Barofsky que Levitin (2014) también menciona, pueden ayudar a crear presión de la opinión pública sobre los políticos. Pero, como Levitin (2014) señala, las regulaciones no son muy sensibles a los procesos democráticos en parte debido a la falta de familiaridad del público con los temas en cuestión. En AH (2013a, b, 2014) y en otros lugares, se trata de llenar ese vacío ayudando a más gente a comprender los argumentos de los banqueros y de los políticos, creando así más voces para la reforma (ver también la charla de 15 minutos en el evento Stanford Tedx del 20 de mayo de 2014 publicado en http://www.youtube.com/watch?v=s_14vx7gHPQ y el material disponible en http://bankersnewclothes.com/ o http://www.gsb.stanford.edu/news/investigación/ admati.etal.html). Este esfuerzo ayuda a enfrentar el desafío de la política financiera y, por lo tanto, no está "fuera de lugar", como Levitin (2014, p. 73) parece implicar.
} 
Las fuerzas económicas discutidas en la Sección III también parecen ser mal interpretadas o subestimadas por los economistas académicos, incluyendo aquellos que se especializan en macroeconomía y en la banca. Esta situación se refleja en los falsos tradeoffs discutidos en la Sección IV, que se utilizan para justificar el statu quo y, así, estimulan su persistencia. Una mejor comprensión de estos temas mejoraría la investigación y la enseñanza, que contribuirían con el diseño y la implementación de una mejor regulación.

\section{Conclusiones}

La industria bancaria está plagada de conflictos de intereses. Los que toman o permiten riesgos excesivos no cargan con la suficiente responsabilidad sobre dichos riesgos y sus consecuencias, y los perjudicados tienen muy poco control (o no se dan cuenta de que están siendo afectados). La gravedad de la crisis financiera de 2007-2009 se debe a las regulaciones deficientes e ineficaces, pero los responsables del fracaso no han aprendido todas las lecciones.

La Ley Dodd Frank en los EE.UU. da mucha autoridad a los reguladores, pero no garantiza que los reguladores utilizarán efectivamente dicha autoridad o que lo harán de manera eficiente. ${ }^{49}$ La situación es similar en otros lugares. Una regulación más fuerte y mejor diseñada para incrementar el uso de capital en la banca traería grandes beneficios. Es más, la regulación de apalancamiento es altamente favorable en términos de costo-beneficio en relación a sus alternativas y podría reducir la necesidad de intervenciones más costosas. Sin embargo, la oportunidad para una reforma importante no se ha materializado hasta ahora.

Los temas que ayudarían a que la regulación de capital fuera más sencilla de implementar y que contribuyera a la estabilidad financiera incluyen: (i) mejorar la divulgación de la información, especialmente en los mercados de derivados, para reducir la opacidad del sistema; (ii) modificar las leyes tributarias para evitar penalizar el fondeo con capital; (iii) reexaminar las amplias exen-

\footnotetext{
${ }^{49}$ Por ejemplo, en el Título I de la Ley, los reguladores deben tomar medidas para reducir el riesgo que las instituciones cuya quiebra podría ser perjudicial generan al público. Sin embargo, y de manera contraproducente, en varias ocasiones la Reserva Federal permitió que la mayoría de ese tipo de instituciones realizara pagos a sus accionistas, repitiendo errores previos a la crisis. Las "pruebas de resistencia" usadas para justificar esta acción no son alentadoras. Ver $\mathrm{AH}$ (2013a, Capítulo 11) para ampliar el debate.
} 
ciones que los derivados y las operaciones de pase tienen en la legislación de quiebra. ${ }^{50,51}$

A diferencia de los automóviles, aviones o puentes inseguros, donde el daño es visible y la rendición de cuentas a menudo puede establecerse, el daño de la toma excesiva de riesgo en el sector bancario es abstracto, y la responsabilidad por tomar o tolerar riesgos excesivos es limitada. Sin embargo, el daño que puede producir la banca puede afectar a toda la economía y el impacto negativo puede ser duradero; y los mercados distorsionados por las externalidades, los subsidios descomunales y las fallas de gobierno no producen resultados eficientes. Los argumentos para una mejor regulación de apalancamiento son fuertes, sin embargo, crear la suficiente voluntad para que los hacedores de política económica se involucren en estos temas y tomen medidas sigue siendo un desafío. ${ }^{52}$

\footnotetext{
50 Ver, por ejemplo, “What's Inside the America's Banks?", Jesse Eisinger y Frank Partnoy, The Atlantic, enero-febrero de 2013. La mayor parte de las operaciones con derivados deberían realizarse en mercados de valores con precios observables públicamente. El episodio London Whale mostró que los riesgos que quedan fuera de la vista de administradores y reguladores pueden ser sustanciales. Los bancos no tienen suficientes incentivos para la administración de los riesgos.

${ }^{51}$ Ver Skeel y Jackson (2012) y "Reforming Repo Rules", Mark Roe, Project Syndicate, 21 de diciembre de 2011.

${ }^{52}$ Los reguladores en la mayoría de los países tienen la suficiente autoridad legal para implementar mejores regulaciones. Algunas otras cuestiones de política requieren cambios en las leyes (por ejemplo, las impositivas o de quiebra) y requieren la coordinación internacional, particularmente en el contexto de los casos de resolución transnacional.
} 


\section{Referencias}

Admati, A. R., P. M. DeMarzo, M. F. Hellwig y P. Pfleiderer (2013). "Fallacies, Irrelevant Facts, and Myths in the Discussion of Capital Regulation: Why Bank Equity is Not Socially Expensive", Documento de trabajo (primera versión de agosto de 2010).

Admati, A. R., P. M. DeMarzo, M. F. Hellwig y P. Pfleiderer (2014). "The Leverage Ratchet Effect", Documento de trabajo.

Admati, A. R., y M. F. Hellwig (2011). "Good Banking Regulation Needs Clear Focus, Sensible Tools, and Political Will", Documento de trabajo.

Admati, A. R., y M. F. Hellwig (2013a). The Bankers'New Clothes: What's Wrong with Banking and What to Do about It, Princeton University Press (extractos y links disponibles en http://bankersnewclothes.com/).

Admati, A. R., y M. F. Hellwig (2013b). "Does Debt Discipline Bankers? An Academic Myth about Bank Indebtedness", Documento de trabajo.

Admati, A. R., y M. F. Hellwig (2014). "The Parade of Bankers' New Clothes Continues: 28 Flawed Claims Debunked”, Documento de trabajo.

Berk, J. y P. DeMarzo (2013). Corporate Finance, Third Edition, Prentice Hall.

Brunnermeier, M., y M. Oehmke (2013). "The Maturity Rat Race", Journal of Finance, Vol. 68, Issue 2, pp. 483-521.

Calomiris, C. W. (2013). "Reforming Banks Without Destroying Their Productivity and Value", Journal of Applied Corporate Finance, Vol. 25 (4), pp. 14-20.

Cochrane, J. H. (2014). "Towards a Run-Free Financial System", Documento de trabajo.

Cole, R. A. (2013). "How did the Financial Crisis Affect Business Lending in the U.S?", Documento de trabajo. 
Diamond, D. W., y R. G. Rajan (2012). "Illiquid Banks, Financial Stability, and Interest Rate Policy", Journal of Political Economy, 120, pp. 552-591.

French, K., M. N. Baily, J. Y. Campbell, J. H. Cochrane, D. W. Diamond, D. Duffie, A. K. Kashyap, F. S. Mishkin, R. G. Rajan, D. S. Scharfstein, R. J. Shiller, Hyun Song Shin, M. J. Slaughter, J. C. Stein, y R. M. Stulz (2010). The Squam Lake Report: Fixing the Financial System, Princeton University Press.

Gordon, J. N. y C. Gandia (2013). "Money Market Funds Run Risk: Will Floating Net Asset Value Fix the Problem?”, Documento de trabajo.

Gorton, G. (2012). Misunderstanding Crises: Why We Don't See them Coming, Oxford University Press.

Heffernan, M. (2012). Willful Blindness: Why We Ignore the Obvious at Our Peril, Walker \& Company.

Haldane, A. G. (2011a). "Capital Discipline”, presentación en las reuniones de la American Economic Association, enero.

Haldane, A. G. (2011b). "Control Rights (and Wrongs)", Wincott Annual Memorial Lecture, London, octubre.

Hellwig, M. F. (2014). "Liquidity Provision and Equity Funding of Banks", Documento de trabajo (próximamente).

Levitin, A. J. (2014). "The Politics of Financial Regulation and the Regulation of Financial Politics: A Review Essay”, Harvard Law Review (próximamente).

Mishkin, F. S. (2013). The Economics of Money and Financial Markets, Business School Edition, 3ra. edición, Pearson.

Modigliani, F., y M. H. Miller (1958). "The Cost of Capital, Corporation Finance, and the Theory of Investment”, American Economic Review, 48, pp. 261-297.

Myers, S. C. (1977). "Determinants of Corporate Borrowing", Journal of Financial Economics, 5, pp. 147-175. 
Myers, S. C. y N. S. Majluf (1984). "Corporate Financing and Investment Decisions when Firms Have Information that Investors Do Not Have", Journal of Financial Economics, 13, pp. 187-222.

Pfleiderer, P. (2010). "On the Relevancy of Modigliani and Miller to Banking: A Parable and Some Observations", Documento de trabajo.

Pfleiderer, P. (2014). "Cameleons: The Misuse of Theoretical Models in Finance and Economics", Documento de trabajo.

Skeel, D. A. Jr., y T. H. Jackson (2012). "Transaction Consistency and the New Finance in Bankruptcy”, Columbia Law Review, pp. 152-202. 